\title{
Microsponge Technology for Innovative Drug Delivery System
}

\author{
Lendave A. S.
}

Department of Pharmaceutics, SVPM's College of Pharmacy, Svitribai Phule Pune University, Malegaon Tal, Baramati Dist- Pune, Maharashtra, India

\section{Article Info}

Volume 7, Issue 5

Page Number: 63-75

Publication Issue :

September-October-2020

\section{Article History}

Accepted : 10 Sep 2020

Published : 20 Sep 2020

\section{ABSTRACT}

Microsponge drug delivery system (MDDS) technology holds a remarkable promise for achieving the aim of controlled and site-specific drug delivery which reduce systemic exposure and minimize local cutaneous reactions to active drug and as a result, has attracted huge interest of researchers. Microsponges consist of microporus beads, typically 10-25 microns in diameter, loaded with active agent. When carried out to the skin, the microsponge releases its active element on a time mode and also in reaction to different stimuli (rubbing, temperature, $\mathrm{pH}$, and many others) which can be used ordinarily for topical and lately for oral management. This article gives a extensive assessment of Microsponges drug transport system discussing the concepts and practise methods. Appropriate analytical techniques for characterization of microsponges like particle size and its distribution, surface morphology, porosity, density, In Vitro drug release studies as well as applications of microsponge and future prospects are covered. Advantages/Potential functions, limitations and their possible remedies of the microsponge and programmable parameters are also mentioned. The microsponge are used in the sunscreens, creams, ointments, over the counter skin care preparations, which are meant for topical application. microsponge drug delivery can provide increased efficacy for topical active agent with enhanced safety, extended product stability.

Keywords: Microsponges, Transdermal Drug Delivery, Programmable Release, Topical Formulation, Applications.

\section{INTRODUCTION}

\section{Topical drug delivery system}

Drug products topically administered via the skin fall into fashionable categories, the ones implemented for nearby movement and those for systematic effects. Local moves encompass the ones at or on the surface of the pores and skin, those that exert their movements on the stratum corneum and people that modulate the function of the dermis and or the epidermis. Common products inside the former class 
include creams, gels, ointments, pastes, suspensions, lotions, foams, sprays, aerosols. The Creams, ointments and gels normally are referred to as semisolid dosage forms. The maximum not unusual drug merchandise carried out to the skin for systemic outcomes are called self adhering transdermal drug delivery systems (TDS) or transdermal patches.

In the modern years the improvement of new tablets isn't always sufficient for the drug treatment. But it also includes the development of suitable drug delivery gadget at site of action. The In Vivo destiny of the drug is not most effective determined by using the residences of the drug, but it additionally determined via the service device, which allows a managed and localized release of the active drug consistent with the particular need of the remedy. The microsponge primarily based polymeric microspheres uniquely triumph over issues associate with above technology. Microsponges are designed to supply pharmaceutical energetic components efficiently on the minimal dose and additionally to decorate stability, reduce facet results and alter drug release. ${ }^{[1,2]}$

\section{Microsponge}

Microsponge drug delivery system (MDDS) generation holds a brilliant promise for accomplishing the aim of managed and placement-spesific drug delivery which lessen systemic publicity and decrease neighborhood cutaneous reactions to energetic drug. Microsponges consist of microporus beads, usually 10 25 microns in diameter, loaded with active agent. When carried out to the skin, the microsponge releases its active aspect on a time mode and also in response to different stimuli (rubbing, temperature, $\mathrm{pH}$, and so on) that are used broadly speaking for topical and recently for oral administration.

Microsponges are microscopic spheres able to absorbing skin secretions, therefore lowering oiliness and shine form the pores and skin. Spherical particles composed of clusters of even tinier sheres are capable of maintaining 4 instances their weight in skin secretions. These merchandise are normally offered to the purchaser in conventional bureaucracy like lotions, gels or creams they include a incredibly high concentration of active factor. $[1,2,3,5,8]$

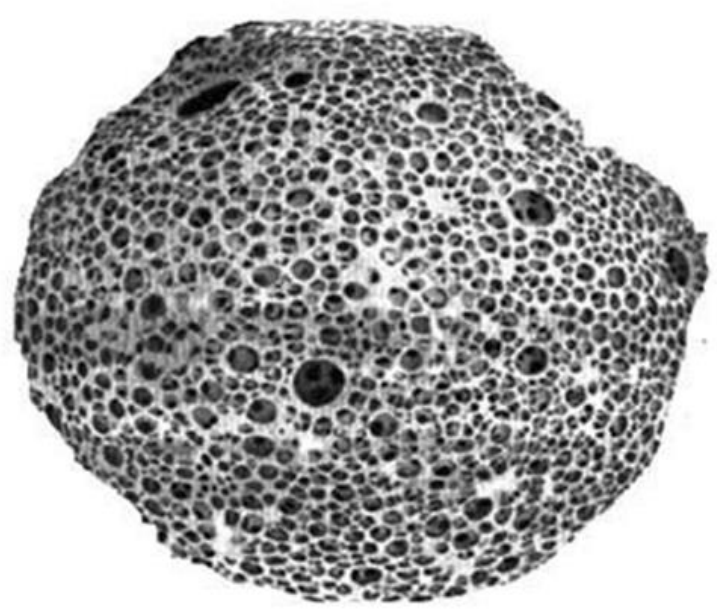

Figure $1:$ Highly porous nature of microsponges History of microsponge

The microsponge generation turned into developed through Won in 1987 and the original patents had been assigned to Advanced Polymer Systems, Inc. This enterprise developed a large numer of versions of the approach and implemented the ones to beauty in addition to OTC and prescription pharmaceutical products. ${ }^{[2,3]}$

\section{Methods of preparation of Microsponges}

The selection of a particular encapsulation approach is ordinarily decided by means of the solubility traits of the drug and polymer. A famous method for the encapsulation of water insoluble drugs within water insoluble polymers is the diffusion solvent method. This technique may be each easily finished inside the laboratory but has scale up capacity such that massive volumes of water may be handled. When subsequently developing a microencapsulation 
manner then in the end decided on method must preferably produce.

$>$ High yields of microparticles and free of massive agglomeration.

$>$ Higher encapsulation of the center material.

$>$ A reproducible launch profile from batch to batch.

$>$ An potential to alter In Vitro release rates by various process parameters with the intention to prepare microparticles with the desired In Vivo release traits.

\section{Preparation strategies are described as follows;}

1) Liquid-liquid suspension polymerization method.

2) Quasi-emulsion solvent diffusion method.

\section{Liquid-liquid suspension polymerization method}

The porous micro spheres are prepared by using suspension polymerization method in liquid-liquid systems. The polymerization procedure leads to the formula of a reservoir type of gadget, which opens on the surface via pores. In a few cases an inert liquid immiscible with water but completely miscible with monomer is used throughout the polymerization to shape the pore network. After the polymerization the liquid is eliminated leaving the porous microspheres, i.e. Microsponges. Impregnating them within carried out microsponges then consists of the functional materials. Sometimes solvent can be used for quicker and green incorporation of the active materials. The microsponges act as topical carriers for sort of practical substance, e.g. Antiacne, anti-inflammatory, anti purities, anti fungal, rubefacients, and so on. Whilst the drug is sensitive to the polymerization conditions, step procedure is used. The polymerization is achieved the usage of substitute porogen and is changed by using the purposeful substance beneath mild experimental condition.

\section{Procedure}

Microsponges are prepared with the aid of suspension polymerization method is liquid-liquid structures. Firstly, the monomers are dissolved together with energetic substances (non-polar drug) in the correct solvent solution of monomer, which can be then dispersed within the aqueous segment with agitation. Aqueous section typically include components along with surfactants and dispersants (postponing marketers) and so on with the intention to facilitate the formation of suspension. Once the suspension is hooked up with awesome droplets of the desired size then, polymerization is initiated by the addition of catalyst or via increasing temperature in addition to irradiation. The polymerization method ends in the development of a reservoir type of machine that opens at the surface via pores. During the polymerization, an inert liquid immiscible with water however completely miscible with monomers is used to shape the network in some instances. Once the polymerization system is completed, the numerous steps concerned inside the preparation of microsponges are summarized as follows;

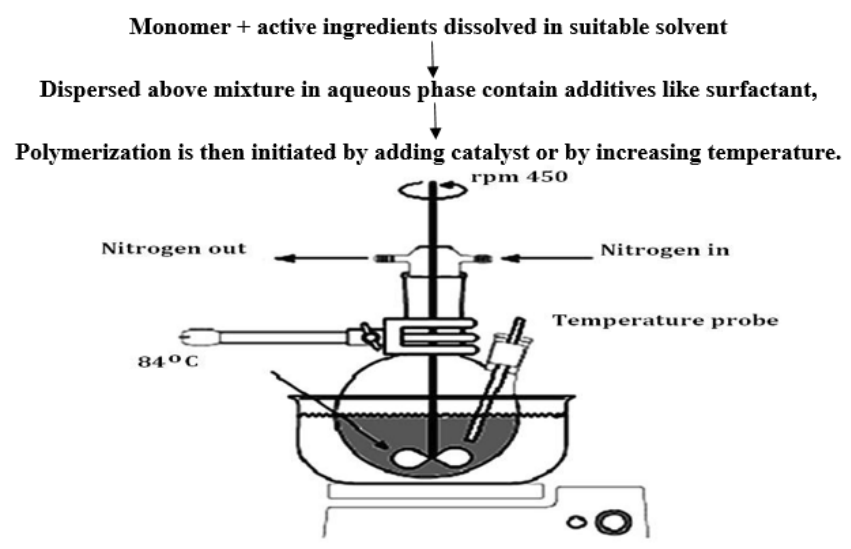

Figure 2 : Microsponge preparation by liquid-liquid suspension polymerization.

\section{Quasi-emulsion solvent diffusion method}

This is Top-down technique beginning with preformed polymer. This method worried formation of quasi-emulsion of two distinctive phase i.e. Inner 
phase and outside phase much like emulsions. The internal phase of drug polymer solution made in a risky solvent like ethanol or acetone or dichloromethane was added to outside section comprising the aqueous polyvinyl alcohol (PVA) solution with full of life stirring. Triethylcitrate (TEC), which was brought at an good enough amount so on $\gg$ can facilitate plasticity. Stirring lead to the formula of discrete emulsion globules referred to as quasi $\rightarrow$ emulsion globules. Solvent become then extracted out from those globules to form insoluble, rigid microparticles i.e. Microsponges. Following enough stirring, the combination turned into then filtered to separate the microsponges. The microsponge then dried in an air heated oven. Conceptually, the finely dispersed droplets of the polymeric solution of the drug (dispersed phase) get solidified in aqueous section via counter diffusion of organic solvent and water out of and into the droplets. The subtle aqueous phase within the droplets decreased the drug and polymer solubility resulting within the co $\rightarrow$ precipitation of both the additives and continued diffusion of the organic segment results in in addition solidification, generating matrix-kind porous microspheres. In assessment with liquid-liquid suspension polymerization technique, this approach supplied the benefit of much less publicity of the drug to the ambient circumstance, low solvent residues in the product because the solvent get extracted out due to its solubility in aqueous media or due to its volatile nature.

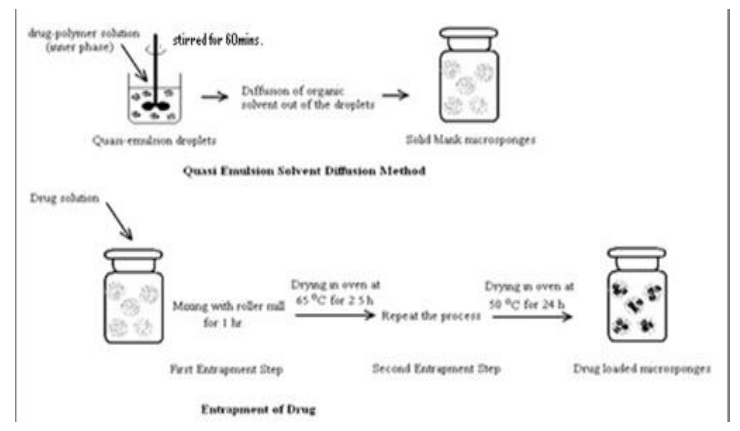

Figure 3 : Preparation of microsponges by quasiemulsion solvent diffusion method
Potential features of Microsponge drug delivery system

Microsponges show ideal balance over $\mathrm{pH}$ ranging from 1 to 11 and at excessive temperature up to $130^{\circ} \mathrm{C}$.

Microsponges show off good compatibility with various vehicles and components.

Microsponges have high entrapment performance up to 50 to $60 \%$.

Microsponges are characterised by way of freeflowing houses.

The average pore size of microsponge is small $(0.25 \mu \mathrm{m})$ in a manner to save you the penetration of bacteria; accordingly they do not need sterilization or addition of preservatives.

Microsponges are non-allergenic, non-aggravating, non-mutagenic and non-poisonous.

Microsponges can absorb oil up to six instances their weight with out drying.

Microsponges can enhance product overall performance.

Microsponges can decreased infection and consequently improve affected person compliance.

Microsponges can enhance product elegancy. ${ }^{[7,8,28]}$

\section{Limitations of Microsponge drug delivery system}

The coaching techniques generally use natural solvents as porogens, which pose an environmental threat, as some may be noticeably inflammable, posing a safety risk. In a few cases, the strains of residual monomers had been found, which can be toxic and unsafe to fitness. ${ }^{[3,5,8]}$

\section{Formulation concerns}

Actives entrapped in MDDS can then be integrated into many merchandises which include lotions, gels, creams, powders and soaps or can be compressed into capsules. When formulating the vehicle, certain considerations are taken into account for you to gain preferred product traits. 
$>$ The solubility of actives inside the vehicle have to be restricted. Otherwise the vehicle will use up the microsponges before the utility.

$>$ To keep away from cosmetic problems; now not greater than 10 to $12 \% \mathrm{w} / \mathrm{w}$ microsponges have to be incorporated into the automobile.

$>$ Polymer layout and payload of the microsponges for the active have to be optimized for required launch price for given time period. $[8,5,30]$

\section{Hypothetical mechanism of action}

The active aspect is introduced to the automobile in an entrapped shape. As the microsponge debris have an open shape (they do now not have a continuous membrane surrounding them), the energetic is free to transport inside and out from the particles and into the vehicle until equilibrium is reached, when the vehicle becomes saturated. Once the completed product is implemented to the pores and skin, the lively that is already within the vehicle could be absorbed into the skin, depleting the vehicle, which will become unsaturated, consequently, stressful the equilibrium. This will start a float of the active from the microsponge particle into the vehicle, and from it to the skin, until the vehicle is either dried or absorbed. Even after that the microsponge debris retained at the surface of the stratum corneum will continue to steadily launch the energetic to the pores and skin, offering extended release over the years. This proposed mechanism of action highlights the significance of formulating motors for use with microsponge entrapments. If the energetic is too soluble inside the preferred automobile throughout compounding of the completed products, the goods will no longer provide the desired advantages of slow launch. Instead they will behave as though the lively changed into introduced to the automobile in a loose from. Therefore, even as formulating microsponge entrapments, it is crucial to layout a vehicle that has minimum solubilizing power for the actives. This principle is opposite to the traditional system ideas generally carried out to topical products. For traditional device it is normally encouraged to maximize the solubility of the lively inside the vehicle. When the usage of microsponge entrapments, a few solubility of the active in the vehicle is suitable, because the vehicle can provide the initial loading dose of the energetic until launch from the microsponge is activated by way of the shift in equilibrium from the polymer into the carrier. Another manner to keep away from undesirable premature leaching of the lively from the microsponge polymer is to formulate the product with a few loose and a few entrapped active, so the vehicle is pre-saturated. In this case there will no longer be any leaching of the lively from the polymer at some stage in compounding. The charge of active release will in the long run rely now not best on the partition coefficient of the energetic element between the polymer and the vehicle (or the pores and skin), but also on some of the parameters that signify the beads. Examples of these consist of floor area and often, mean pore diameter. Release can also be managed via diffusion / different triggers including moisture, $\mathrm{pH}$, friction or temperature. $[9,10,29]$

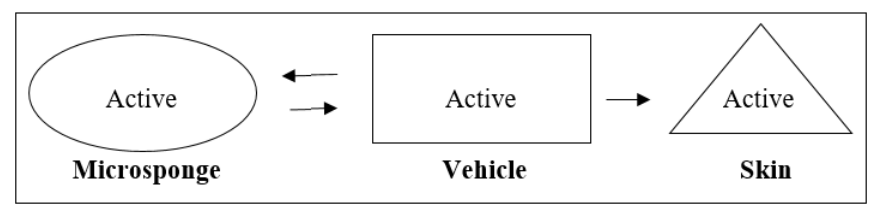

Figure 4 : schematic representation of the distribution of the loaded material (active) on skin

\section{Release mechanism}

The mentioned programmable parameters may be efficiently manipulated to design microsponge transport device for the release of useful substance over a time period in reaction to one or extra outside 
stimuli. The launch mechanism of this system is specially as observe;

\section{A. Sustained or time release}

In the development of a sustained release microsponge, unique bodily and chemical parameters of the entrapped energetic substance along with volatility, viscosity and solubility will be studied while in case of polymeric microsponge pore diameter, quantity, and resiliency of the polymeric microspongd. are evaluated to present important sustained launcl2. results.

\section{B. Release on command}

Microsponge may be designed to release the given amount of lively components over time in response to one or greater outside triggers.

\section{Pressure launch}

Microsponge device launch fluid or active factor when it is pressed or squeezed, there by means of replenishing the level of entrapped energetic aspect onto the skin. The amount released may depend on the release of the sponge and the resiliency of the microsponge.

\section{Temperature release}

The release of lively ingredients from microsponge can be activeated with the aid of temperature. At room temperature, few entrapped active ingredients may be too viscous to glide unexpectedly from microsponge onto the skin. With boom in skin temperature, drift fee is likewise multiplied and therefore release is likewise superior.

\section{PH based totally release}

Triggering the $\mathrm{pH}$-primarily based release of the lively may be accomplished by editing the coating at the microsponge. $\mathrm{PH}$ have many utility in drug delivery.

\section{Solubility based release}

Microsponges loaded with water miscible ingredients like antiseptics and antiperspirants will launch the aspect in the presence of water. The launch can be activated by means of diffusion but deliberating, the partition coefficient of the factor between the microsponges and the outside machine. ${ }^{[11,4,5,29,30]}$

\section{Characterization of microsponge}

\section{Particle size and distribution analysis}

Particle size analysis of loaded and unloaded microsponges can be performed by laser light diffractometry. The values (d50) can be expressed for all formulation as mean size range. As well as the particle size was determined using an optical microscope. The microscope was fitted with a stage micrometer to calibrate the eyepiece micrometer. The values were given for the form of mean particle size range.

One division of stage micrometer $=0.01 \mathrm{~mm}=10 \mu \mathrm{m}$

$$
\mathrm{C}=\frac{\mathrm{SM} \times 10}{\mathrm{EM}}
$$

Where,

\section{$\mathrm{C}=$ correction factor;}

$\mathrm{SM}=$ reading of stage micrometer which coincides with reading of eyepiece micrometer (E.M.).

The particle diameter of around 100 particles was measured. The average particle size was determined using the following formula:

$$
\text { D mean }=\frac{\sum \mathrm{nd}}{\sum \mathrm{n}}
$$

Where, $\mathrm{n}=$ number of microsponges observed.

$\mathrm{d}=$ mean size range.

Each formulation was observed three times and average of three trials was calculated. Particle size 
larger than $30 \mu \mathrm{m}$ can impart gritty feeling and hence particles of sizes between 10 and $25 \mu \mathrm{m}$ are preferred to use in final topical formulation.

\section{Morphology and surface topography}

For morphology and surface topology, prepared microsponges can be coated with gold-palladium under an argon atmosphere at room temperature and then the surface morphology of the microsponges can be studied by scanning electron microscopy (SEM). SEM of a fractured microsponge particle can also be . taken to illustrate its ultrastructure.

\section{Production yield}

For calculating production yield, theoretical mass was calculated initially by taking the weight of excipients8. The production yield of the microsponges was determined accurately by calculating the initial weight of the excipients ad the final weight of the formulation obtained. The production yield was calculated In triplicate using the following equation.

Production yield

$=\frac{\text { Particle mass of microsponges }}{\text { Theoretical mass }} \times 100$

\section{Drug entrapment efficiency}

The entrapment efficiency and drug loaded microsponges were estimated by dissolving $50 \mathrm{mg}$ of microsponges in $0.01 \mathrm{~N}$ HCL. The sample were analyzed using UV-visible spectrophotometer efficiency was calculated a triplicate using the following equation.

Drug entrapment efficiency (\%) = Weight of drug in microspheres $\backslash$ weight o drug fed initially x 100

\section{In Vitro drug release studies}

The drug release studies of drug loaded microsponges were carried out using basket dissolution apparatus (USP type I apparatus).900ml of fresh phosphate buffer saline (PBS pH5.5 to 7.4) was used for the release studies. The temperature of the dissolution medium was controlled at $32 \pm 1^{\circ} \mathrm{C}$ with $150 \mathrm{rpm}$ rotation speed. Microsponges equivalent to $50 \mathrm{mg}$ were weighed. At fixed time intervals, aliquots of samples were withdrawn and replaced by an equal volume of fresh dissolution medium to maintain the sink condition. After suitable dilution, the samples were analyzed spectrophotometrically.

\section{Determination of true density}

The true density of microsponge can be measured using an ultra-pycnometer under helium gas and is calculated from a mean of repeated determinations.

\section{Characterization of Pore Structure}

Pore volume and diameter are vital in controlling the intensity and duration of effectiveness of the active ingredient. Pore dimeter also affects the migration of active ingredients from microsponges into the vehicle in which the material is dispersed. Mercury intrusion porosimetry can be employed to study effect of pore diameter and volume with rate of drug release from microsponges.

Porosity parameters of microsponges include intrusion extrusion isotherms. Pore size distribution, total pore surface area, average pore diameters, shape and morphology of the pores, bulk and apparent density can be determined by using mercury intrusion porosimetry. Incremental intrusion volume scan be plotted against pore diameters that represented pore size distributions. The pore diameter of microsponges can be calculated by using;

Washburn equation:

$$
\mathrm{D}=-\frac{4 \gamma \cos \theta}{\mathrm{P}}
$$

Where $D$ is the pore diameter $(\mu \mathrm{m}) ; \gamma$ the surface tension of mercury (485 dyn $\mathrm{cm}^{-1}$ ); $\theta$ he contact angle $\left(130^{\circ}\right)$; and $\mathrm{P}$ is the pressure (psia). 
Total pore area (Atot) was calculated by using equation,

$$
\text { Atot }=\frac{1}{\gamma \cos \theta} \int_{0}^{V t o t} P \cdot d v
$$

Where $\mathrm{P}$ is the pressure (psia); $\mathrm{V}$ volume $\left(\mathrm{ml}^{-1} \mathrm{~g}^{-1}\right) ; \mathrm{V}_{\text {tot }}$ is the total specific intrusion volume $\left(\mathrm{ml} \mathrm{g}^{-1}\right)$. The average pore diameter $(\mathrm{Dm})$ was calculated by using following equation.

$$
\mathrm{Dm}=\frac{4 \mathrm{Vtot}}{\text { Atot }}
$$

Envelope (bulk) density (pse) of the microsponges was calculated by using equation,

$$
\text { Pse }=\frac{\text { Ws }}{V p-V h g}
$$

Where Ws is the weight of the SPM sample (g); Vp the empty penetrometer $(\mathrm{ml})$; Vhg is the volume of mercury (ml). absolute (skeletal) density (nas) of microsponges was calculated by using equation,

$$
\text { Pse }=\frac{\text { Ws }}{\text { Vp-Vtot }}
$$

Where Vse is the volume of the penetrometer minus the volume of the mercury $(\mathrm{ml})$.

Finally, the \% porosity of the sample was found from equation,

$$
\text { Porosity }(\%)=\left(1-\frac{\text { Pse }}{\text { Psa }}\right) \mathrm{X} 100
$$

Pore morphology can be characterized from the intrusion-extrusion profiles of mercury in the microsponges. [6, 8, 12, 13, 14, 28]

\section{Applications of microsponge}

The merchandise below improvement or in the market region make use of the Topical Microsponge Drug Delivery Systems in three number one methods

1. As reservoirs releasing lively components over an extended time frame.

2. As receptacles for absorbing unwanted substance, inclusive of extra skin oils.

3. As closed packing containers preserving substances away from the skin for superficial movement.

The common lively aspect in traditional products is found in a exceedingly high concentration and, while implemented to the skin, may be unexpectedly absorbed. The common end result is overmedication, accompanied by way of a length of under remedy till the following software. Rashes and more critical side results can arise while the active ingredients rapidly penetrate under the pores and skin floor. Microsponge generation is designed to permit a extended charge of launch of the active elements, there by means of presenting potential discount inside the aspect consequences at the same time as preserving therapeutic efficacy. ${ }^{[8,14,15,30]}$

\section{In topical drug delivery}

The benzoyl peroxide (BPO) is commonly utilized in topical formulations for the treatment of acne and athletes foot. Skin irritation is a commonplace aspect effect, and it has been proven that managed release of BPO from a delivery gadget to the pores and skin may want to lessen the facet impact while lowering percutaneous absorption. BPO micro-debris had been organized using an emulsion solvent diffusion approach by means of adding an organic internal phase containing benzoyl peroxide, ethyl cellulose and dichloromethane right into a stirred aqueous 
section containing polyvinyl alcohol. Disorders of hyper pigmentation including melasma and submit inflammatory hyper pigmentation (PIH) are not unusual, in particular amongst human beings with darker skin sorts. Hydroquinone (HQ) bleaching lotions are taken into consideration the gold widespread for treating hyper pigmentation. Recently new method of HQ $4 \%$ with retinol $0.15 \%$ entrapped in microsponge reservoirs become advanced for the remedy of melasma and PIH. Microsponges were used to launch HQ steadily to prolong publicity to remedy and to limit skin infection. ${ }^{[3,16]}$

\section{In oral drug transport}

A microsponge system gives numerous benefits for oral drug delivery consisting of

- Preserve the lively elements within a protected environment and provide oral controlled delivery to the lower part of the gastrointestinal tract (GIT).

- Microsponge systems improve the solubility of poorly soluble capsules by using entrapping these pills of their porous structure.

- As the porous structure of the microsponge is very small in size, the drug entrapped might be reduced to microscopic debris with better floor vicinity, and consequently advanced price of solubilization.

- Maximize the quantity of drug to be absorbed, because the time it takes the microsponge device to bypass thru the gut is appreciably elevated.

Ketoprofen become used as a model drug for systemic drug delivery of microsponges inside the have a look at. Ketoprofen microsponges had been organized with the aid of quasi-emulsion solvent diffusion method with Eudragit RS a hundred and afterwards tablets of microsponges had been organized with the aid of direct compression technique. Different stress values were carried out to the tablet powder mass to be able to determine the most appropriate strain valve for compression of the tablet. Results indicated that compressibility became an awful lot progressed over the bodily mixture of the drug and polymer; due to the plastic deformation of sponge-like structure, microsponges produce mechanically robust pills. $[17,3$, 23]

\section{In bone tissue engineering}

3D biodegradable porous scaffold plays a completely crucial position in articular cartilage tissue engineering. The hybrid structure of three-D scaffolds turned into advanced that mixed the blessings of herbal type 1 collagen and synthetic PLGA knitted mesh. The mechanically robust PLGA mesh served as a skeleton whilst the collagen microsponges facilitated mobile seeding and tissue system.

The scaffolds were divided into three groups:

a. THIN: collagen microsponge fashioned in interstices of PLGA mesh;

b. SEMI: collagen microsponge fashioned on one aspect of PLGA mesh;

c. SANDWICH: collagen sponge shaped on both aspects of PLGA mesh.

Bovine chondrocytes have been cultured in these scaffolds and transplanted subcutaneously into nude mice for 2, 4, and 8. All 3 groups of transplants showed homogeneous cellular distribution, herbal chondrocyte morphology, and ample cartilaginous ECM deposition. Production of GAGs in step with DNA and the expression of kind II collagen and aggrecan mRNA had been tons better within the SEMI and SANDWICH organization than inside the THIN institution. When in comparison to native articular cartilage, the mechanical strength of the engineered cartilage reached 54.8\%, 49.3\% in Young's modulus and $68.8 \%, 62.7 \%$ in stiffness, respectively, in SEMI and SANDWICH. These scaffolds may be 
used for the tissue engineering of articular cartilage with adjustable thickness. The design of the hybrid systems offers a strategy for the practise of 3 -D porous scaffolds. A novel 3-dimensional porous scaffold has been advanced for bone tissue engineering by way of hybridizing synthetic poly (DL-lactic-co-glycolic acid) (PLGA), naturally derived collagen and inorganic apatite. First, a porous PLGA sponge become organized. Then collagen microsponge were shaped within the pores of the PLGA sponge. Finally, apatite particulates have been deposited on the surfaces of the collagen microsponges in the pores of PLGA sponge. The deposited particle has been small and scarce after one cycle of change immersion. Their variety and length elevated with the variety of trade immersion cycles. The surface of collagen microsponges have been absolutely blanketed with apatite after three cycles of alternate immersion. The porosity of the hydrid sponge described steadily because the number of change immersion increased. The deposition became controllable. Use of the PLGA sponge as a mechanical skeleton facilitated formation of the PLGA collagen apatite hybrid sponge into favored shapes and collagen microsponges facilitated the uniform deposition of apatite particulates through out the sponge. The PLGA collagen apatite hybrid sponge might function a useful three-dimensional porous scaffold for bone tissue engineering. ${ }^{[18,19,22]}$

\section{In cardiovascular engineering}

A biodegradable fabric with autologous mobile seeding requires a complex and invasive method that consists of the hazard of contamination. To avoid these problems, a biodegradable graft material containing collagen microsponges that would permit the regeneration of autologous that could allow the regeneration of autologous vessel tissue has evolved. The potential of this fabric to accelerate in situ cellularization with autologous endothelial and smooth muscle mobile turned into tested with and with out precellularization. Poly (lactic-co-glycolic acid) as a biodegradable scaffold turned into compounded with collagen microsponge to shape a vascular patch material. These poly (lactic-co-glycolic acid) collagen patches with $(n=10)$ or without $(n=10)$ autologous vessel cellularization were used to patch the dog pulmonary artery trunk. Histologic and biochemical assessments had been done 2 and 6 month after the implantation. There was no thrombus formation in either institution, and the poly (lacticco-glycolic acid) scaffold changed into nearly completely absorbed in both corporations. Histologic outcomes confirmed the formation of an endothelial cellular monolayer, a parallel alignment of smooth muscle cells, and reconstructed vessel wall with elastin and collagen fibers. The cell and extracellular additives in the patch had multiplied to ranges similar to thouse in native tissue at 6 month. This patch shows promise as a bioengineered material for selling in situ cellularization and the regeneration of autologous tissue in cardiovascular surgery. ${ }^{[20,25]}$

\section{In reconstruction of vascular wall}

The tissue-engineered patch turned into fabricated by means of compounding a collagen-microsponge with a biodegradable polymeric scaffold composed of polyglycolic acid knitted mesh, reinforced on the out of doors with woven polylactic acid. Tissueengineered patches with out precellularization were grafted into the porcine descendnding aorta $(n=5)$, the porcine pulmonary arterial trunk $(\mathrm{n}=8)$, or the canine proper ventricular outflow tract (as the massive graft model; $\mathrm{n}=4$ ). Histologic and biochemical tests were performed1, 2, and 6 month after the implantation. There was no thrombus formation in any animal. Two months after grafting, all of the grafts showed suitable in situ cellularization through hematoxylin/eosin and immunostaining. The quantification of the cellular population by way of polymerase chain response confirmed a large number of endothelial and clean muscle cells 2 month after implantation. In the large graft version, the structure 
of the patch became just like that of local tissue 6 month after impliantation and this patch may be used as a novel surgical material for the repair of the cardiovascular device. [21, 23, 24]

\section{Future prospects}

Microsponge drug delivery device holds a promising possibility in numerous pharmaceutical applications in the approaching destiny as it has particular homes like stronger product performance and elegancy, prolonged launch, improved drug launch profile, reduced infection, progressed bodily, chemical and thermal balance which makes it flexible to increase novel product forms. The actual challenge in destiny is the improvement of the delivery device for the oral peptide delivery by means of various ratio of polymers. The use of bioerodible and biodegradable polymers for the drug transport is allowing it for the safe delivery of the energetic cloth. As these porous structures have also been studied for the drug delivery via pulmonary direction which indicates that those system can show effective drug launch even within the scarce of the dissolution fluid thus colon is an effective site for concentrated on for drug release. These companies also require to be advanced for opportunity drug management routes like parenteral and pulmonary direction. These debris can also be used because the cell way of life media and therefore also can be hired for stem cell subculture and mobile regeneration in the frame. Due to their beauty, these carrier systems have also found their application in cosmetics. These developments enabled researchers to utilize them variably. These novelties in components additionally open new approaches for drug transport. $[26,27]$

\section{CONCLUSION}

As formulators bear in mind new and innovative ways to supply actives, they can realize the whole talents of these precise substances offering more desirable safety, progressed stability, decreased facet consequences from actives, stronger multifunctionality and progressed element compatibility. Complemented through novel improvement methods and creative formulation strategies, microsponge delivery system may be triumphing strategy for a brand new generation of pharmaceutical and cosmetic industry. Microsponge drug delivery system (MDDS) era holds a fantastic promise for achieving the goal of managed and location-specific drug delivery which lessen systemic publicity and reduce nearby cutaneous reactions to lively drug and as a result, has attracted huge attention of researchers. The microsponge are used inside the sunscreens, creams, ointments, over-the-counter skin care arrangements, which are intended for topical utility. Microsponge drug delivery can offer expanded efficacy for topical active agent with stronger protection, extended product balance.

\section{REFERENCES}

[1]. Patil S, Dandekar V, "Microsponge Drug Delivery System”. European journal of pharmaceutical and medical research.ejpmr, 2010, 3(6), 212-221.

[2]. Jadhav N, Patel V, Bhamare G, Karpe M, "Microsponge delivery system: An updated review, current status and future prospects". Journal of scientific and innovative research 2013; 2 (6): 1097- 1110.

[3]. A. N. Misra., Transdermal Drug delivery, Chapter 5, In: Controlled and Novel Drug Delivery, Edited by N. K. Jain., (1997): 104 105.

[4]. Emanuele A. D., Dinarvand R., Preparation, Characterization and Drug Release from Thermo Responsive Microspheres, Int J Pharm., (1995): 237-242.

[5]. Sharma P.et.al, "Formulation and evaluation of gel-loaded microsponge of roxithromycin for 
topical drug delivery". IOSR Journal of pharmacy (IOSPHR), voi.9, no. 5, 2019, pp.1422.

[6]. Raymond C Rowe, Paul J. Sheskey, Sia^n C. Owen. "Handbook of Pharmaceutical Excipients"5th ed.2006, page no.1-889.

[7]. Rong-Kunchang, Anre Raw, Robert Lionberger, and Lawrence Yu,"Generic development of topical dermatologic products: formulation development, process development, and testing of topical dermatologic products". AAPS J. 2013 Jan; 15(1): 41-52.

[8]. Shaha V, Jain H, Jethva K. "Microsponge drug delivery". Int. J. Res. Pharm. Sci. 2010; 1(2): 212-218.

[9]. Martin A. et. al, In: Physical Pharmacy Physical Chemical Principles in Pharmaceutical Sciences. 3rd ed. 1991;527

[10]. Embil K., Nacht S. The Microspnge Delivery System (MDS): Atopical delivery system with reduced irritancy incorporating multiple triggering mechanisms for the release of actives. J. Microencapsul. 1996; 3(5):575-588.

[11]. Kaity S., Maiti S., Ghosh A., Microsponges: A novel strstegy for drug delivery system. J Adv Pharma Technol Res. 2010; 1(3):283-290.

[12]. Poornima Pandey, Vikas Jain, S. C. Mahajan,. A review: microsponge drug delivery system. Int J Biopharm 2013; 4:225-230.

[13]. Emanuele A. D. et. al, Preparation, characterization and drug release from thermo responsive microspheres. Int . Journal of pharmaceutics. 1995; 237-242.

[14]. Kilicarslan M., Baykara T., The effect of the drug\polymer ratio on the properties of verapamil HCL loaded microspheres. Int. J. Pharm. 2003; 252:99-109.

[15]. Washburn E. W. Note on a method $f$ determining the distribution of pore sizes in a porous material. Proc Natl Acad Sci. 1921; 7(4): 115-116.
[16]. Orr Jr. Application of mercury penetration to material analysis. Powder. Technol. (1969);3: 117-123.

[17]. Neelam Jain, Pramod Kumar Sharma,. Recent advances on microsponge delivery system. Int J Pharm Sci Rev Res 2011; 8:16-22.

[18]. Juni K, Nakano M,. Preparetion and evaluation in vitro of polylactic acid microsphers containing local anesthetic. Chem. Pharma Bull 1981; 29:3363-8.

[19]. Jain Sanjay, Jain N. K. 'The microsponge'. Eastern Pharmacist; 1996:49-53.

[20]. Amrutiya N, Bajaj A, Madan M,. Development of microsponges for topical delivery of mupirocin. AAPS PharmSciTech 2009; 10:402409.

[21]. Comoglu T, Savaser A, Ozkan y, GonulN,. Enhancement of ketoprofen bioavailability formation of microsponge tablets. Phrmazie 2007; 62:51-54.

[22]. Saroj Kumar Pradhan. Microsponge as the versatile tool for drug delivery system. Int J Res Pharm Chem 2011; 1:243-258.

[23]. Cross S. E., Roberts M. S., Subcutaneous Absorption Kinetics of Interferon and Other Solutes, J Pharm Pharmacol., (1993); 45: 606609.

[24]. Elias P. M., Epidermal Lipids, Barrier Function and Desquamation, J Invest Dermatol., (1983); 80: 44-49.

[25]. Dees P. J., Polderman J., Mercury Porosimetry in Pharmaceutical Technology, Powder Technol., (1981); 29: 187-197.

[26]. Fincham J. E, Karnik K. A, Patient counseling and derm therapy. US Pharm 1994; 19:56-57, 61-62, 71-72, 74, 77-78, 81-82.

[27]. Srivastava R, Pathak K,. Microsponges: a futuristic approach for oral drug delivery. Expert Opin. Drug Deliv. 2012; 9(7):863-878.

[28]. D'souza J. I., More H. N., The Microsponge Drug Delivery System: for Delivering 
[29]. an Active Ingredient by Controlled Time Release; Pharmainfo net., (2008); 6 (3):62.

[30]. Sergio Nacht and Martin Katz., The Microsponge: A Novel Topical Programmable Delivery System, Chapter 15, In: Topical Drug Delivery Systems, Edited by David W Osborne and Anfon H. A., (1992); Volume 42: 299 - 325.

[31]. Vikas Jain., Ranjit Singh., Development and Characterization of Eudragit Rs 100 Loaded Microsponges and Its Colonic Delivery Using Natural Polysaccharides, Acta Poloniae Pharmaceutica-Drug Research, (2010); 67: 407415.

\section{Cite this article as :}

Lendave A. S., "Microsponge Technology for Innovative Drug Delivery System", International Journal of Scientific Research in Science and Technology (IJSRST), Online ISSN : 2395-602X, Print ISSN : 2395-6011, Volume 7 Issue 5, pp. 63-75, September-October 2020. Available at doi : https://doi.org/10.32628/IJSRST207521

Journal URL : http://ijsrst.com/IJSRST207521 